\title{
Atrofia muscular espinhal tipo I: aspectos clínicos e fisiopatológicos
}

\author{
Spinal muscular atrophy type I: clinical and pathophysiological aspects
}

\author{
Lucas Rossato Chrun ${ }^{1}$, Larissa Rossato Chrun Costa ${ }^{2}$, \\ Gilson da Silva Miranda ${ }^{1}$, Felipe Monteiro Almeida ${ }^{1}$
}

Chrun LR, Costa LRC, Miranda GS, Almeida FM. Atrofia muscular espinhal tipo I: aspectos clínicos e fisiopatológicos / Spinal muscular atrophy type I: clinical and pathophysiological aspects. Rev Med (São Paulo). 2017 out.-dez.;96(4):281-6.

\begin{abstract}
RESUMO: Objetivo: Realizar uma revisão bibliográfica sobre a etiologia, diagnóstico, tratamento e perspectivas futuras sobre a atrofia muscular espinhal tipo I (doença de Werdnig-Hoffmann). Métodos: Utilizando o banco de dados Medline, Lilacs e Scielo, os artigos mais relevantes foram selecionados, por meio das palavras-chave: atrofia muscular espinhal; atrofia muscular espinhal infantil; doenças neuromusculares; doenças da medula espinhal; doença de Werdnig-Hoffmann. Resultados: Foram localizados 68 artigos, e após análise, 43 estudos, que abordavam os aspectos clínicos e/ou fisiopatológicos, foram selecionados. Discussão: A doença de Werdnig-Hoffmann, atrofia muscular espinhal tipo I, é uma doença hereditária caracterizada pela atrofia e fraqueza muscular progressiva, resultando em elevada e precoce mortalidade. As atrofias musculares espinhais são doenças neuromusculares caracterizadas pela degeneração dos motoneurônios inferiores e são transmitidas seguindo o padrão mendeliano autossômico recessivo. Suas manifestações clínicas são: hipotonia, atrofia, debilidade muscular e diminuição ou ausência dos reflexos osteotendinosos. O tratamento inclui medidas de suporte e medicação específica, o Nusinersen (Spinraza ${ }^{\circledR}$ ), administrado por via intratecal, e pode retardar ou até impedir a evolução da doença, caso seja iniciado precocemente. Conclusão: A doença tem características semelhantes na maioria dos casos e evolui de forma grave. Entretanto, o tratamento emergencial e intensivo, incluindo a medicação específica e atuação de equipe multidisciplinar, pode redefinir e prolongar a sobrevida dos pacientes.
\end{abstract}

Descritores: Atrofia muscular espinal/fisiopatologia; Atrofias musculares espinais da infância/fisiopatologia;Doenças neuromusculares; Doenças da medula espinal/fisiopatologia.

\begin{abstract}
Objective: To produce a literature review about etiology, diagnosis, treatment and future perspectives around spinal muscular atrophy type I (Werdnig-Hoffmann disease). Methods: Using databases like Medline, Lilacs and Scielo; the most important articles about this issue were chosen, using the keywords: spinal muscular atrophy; spinal muscular atrophy of childhood; neuromuscular diseases; spinal cord diseases; Werdnig-Hoffmann disease. Results: 68 articles were collected. After analysis process, 43 studies which focus on the clinical and/or pathophysiological aspects, were selected. Discussion: Werdnig-Hoffmann disease, the spinal muscular atrophy type $\mathrm{I}$, is a hereditary disorder that causes progressive hypotony and muscular weakness. This process evolves with the loss of essential functions, resulting in high and early mortality. The spinal muscular atrophies are neuromuscular diseases characterized by degeneration of lower motor neurons. They are transmitted following the pattern mendelian autosomal recessive. The clinic signs are: hypotony, atrophy, muscular weakness and decrease or absence of osteotendinous reflexes. Treatment includes supportive care and specific medication, Nusinersen (Spinraza $\left.{ }^{\circledR}\right)$, administered by intrathecal injection, and can delay or even stop disease progression if started early. Conclusions: The sickness has similar features on most cases and worsens sorely. However, the intensive and immediate treatment, with the specific medication and the multidisciplinary team - can promote a better quality of life to patients.
\end{abstract}

Keywords: Muscular atrophy, spinal/physiopathology; Spinal muscular atrophies of childhood/physiopathology; Neuromuscular diseases; Spinal cord diseases/physiopathology.

1. Universidade do Estado de Mato Grosso - UNEMAT, Departamento de Medicina, Cáceres, MT, BR. Graduandos em Medicina UNEMAT. E-mails: rc.luca@hotmail.com; gilsonsmiranda@yahoo.com.br; felipemonteiro0714@hotmail.com.

2. Hospital Pequeno Príncipe, Curitiba, PR, BR. Médica Intensivista Pediátrica. E-mail: lary_rc@yahoo.com.br.

Endereço para correspondência: Lucas Rossato Chrun. Rua dos Aviadores, 163, Apto 06. Bairro Santos Dumont. Cáceres, MT, BR. CEP: 78200-000. E-mail: rc.luca@hotmail.com 


\section{INTRODUÇÃO}

\begin{abstract}
Atrofia Muscular Espinhal (AME) tipo I, ou doença de Werdnig-Hoffmann é uma doença neuromuscular de origem genética, autossômica recessiva, que resulta em atrofia muscular progressiva e fraqueza ${ }^{1,2}$.

É causada por baixos níveis da proteína de sobrevivência do neurônio motor (SMN) devido à mutações na decodificação do gene $\mathrm{SMN}-1^{3}$, localizado no cromossomo $5 \mathrm{q} 13^{4,5}$. As alterações mais comumente encontradas são deleções homozigóticas, que são responsáveis pela principal característica fisiopatológica da doença: a degeneração dos neurônios motores inferiores, localizados no corno anterior da medula espinhal ${ }^{2,6}$.

O gene SMN-2 é uma cópia quase idêntica do SMN1, exceto pela substituição de $\mathrm{C}$ (citosina) por $\mathrm{T}$ (timina) na posição 6 do exon $7^{2,6}$. Essa diferença faz com que o gene SMN-2 produza somente uma pequena quantidade de proteína SMN biologicamente ativa, que é o suficiente para a sobrevivência da maioria das células somáticas, com exceção dos neurônios motores ${ }^{3,7}$.
\end{abstract}

Os sintomas podem ter início precocemente, ainda no período pré-natal, quando se observa diminuição dos movimentos fetais, ao nascimento ou nos primeiros seis meses de vida, evoluindo com paralisia muscular progressiva e simétrica, sendo esta mais proximal do que distal ${ }^{8,9}$.

O controle da coluna cervical é comprometido de tal forma que os pacientes têm dificuldade em sustentar a cabeça e não conseguem sentar sem auxílio ${ }^{3,9}$. A tosse e o choro são fracos e, antes de completarem 1 ano de idade, estas crianças perdem a capacidade de deglutir e de se alimentar, desenvolvendo quadro de desnutrição. Todavia, o que as leva à mortalidade em tenra idade são as complicações respiratórias consequentes da disfunção bulbar8. Apesar das afecções motoras, a sensibilidade é preservada, assim como o controle ocular e a visão, o que permite que os movimentos dos olhos sejam usados para interação e comunicação ${ }^{1}$.

Todos os músculos são acometidos pelo processo de atrofia neurogênica, com exceção do diafragma, os músculos das extremidades e os músculos oculares. Apresentam respiração diafragmática paradoxal e com pouca expansibilidade torácica ${ }^{10}$. Ocorre a rápida instalação de contraturas musculares, que acometem os músculos adutores das coxas e rotadores internos dos ombros, conferindo-lhes atitude característica e levando às deformidades torácicas, como o tórax em forma de $\operatorname{sino}^{11}$.

Diante do exposto, e considerando que esta é uma doença neurodegenerativa progressiva, é de fundamental importância que os profissionais da área da saúde tenham condições de fazer um rápido diagnóstico e proporcionar um suporte terapêutico correto e oportuno. Nesse sentido, este trabalho teve como objetivo produzir uma revisão bibliográfica sobre os conhecimentos atuais que versam sobre a etiologia, diagnóstico, tratamento e perspectivas futuras relacionadas à atrofia muscular espinhal tipo I.

\section{MÉTODOS}

Para esta revisão descritiva, foram utilizadas as bases de dados: Medline (www.medline.com/nlm.nih. gov/PubMed); Lilacs (www.bireme.br) e SciELO (www. scielo.br). Foram usados os seguintes descritores: Atrofia muscular espinhal; Atrofia muscular espinhal infantil; Doenças neuromusculares; Doenças da medula espinhal; Doença de Werdnig-Hoffmann.

\section{RESULTADOS E DISCUSSÃO}

Foram encontrados 68 artigos, e após processo de análise, 43 estudos, que abordavam os aspectos clínicos e/ ou fisiopatológicos, foram selecionados.

A principal forma de neuropatia, na infância, é a atrofia muscular espinhal (AME), uma doença neurodegenerativa grave de herança autossômica recessiva, que possui grande variabilidade quanto à época de início e gravidade do comprometimento motor ${ }^{12}$. Ocorre em, aproximadamente, $1: 6000$ a $1: 10000$ nascidos vivos ${ }^{3,4,6,13}$ e sua frequência de portador varia entre $1 / 40$ a $1 / 100$ em diferentes grupos étnicos, sendo mais comum em caucasianos ${ }^{14}$.

O sinal clínico mais característico é a hipotonia muscular ${ }^{15}$, que geralmente tem início nos membros superiores, atingindo posteriormente os membros inferiores e, também, os músculos bulbares. Com a evolução da doença surgem: paresia, amiotrofia, arreflexia e fasciculações ${ }^{16}$.

De acordo com a capacidade funcional e com o prognóstico, a AME é classificada em quatro fenótipos ${ }^{1,5}$. Na AME tipo I, ou doença de Werdnig-Hoffmann, a evolução natural é o óbito antes dos 2 anos de idade em mais de $80 \%$ dos $\operatorname{casos}^{5}$, geralmente por insuficiência respiratória, ao menos que sejam instituídas medidas de suporte ventilatório. ${ }^{3}$ Os primeiros sintomas são observados antes dos 6 meses de vida, quando a criança não consegue manter-se sentada devido à atrofia ${ }^{1,5}$.

Na AME tipo II, os pacientes conseguem sentar sem auxílio, porém ficam impossibilitados de deambular, com surgimento dos primeiros sinais entre 6 e 18 meses $^{1,5}$. No tipo III o paciente consegue andar sozinho, apesar de alguma dificuldade, e na maioria dos casos atinge uma expectativa de vida normal. Por último, no tipo IV, o quadro inicia-se na fase adulta de modo mais brando ${ }^{1,3,5}$.

As alterações genéticas responsáveis pela AME são complexas e envolvem eventos de duplicação, deleção e transição intragênica no gene SMN-1. As formas mais brandas da enfermidade se associam com deleções menores $^{17,18}$. Em 95 a $98 \%$ dos casos, ocorre uma deleção homozigótica no exon 7, e em 2 a 5\% é encontrada uma deleção heterozigótica e uma mutação $0^{5}$. O gene SMN-2, 
devido a substituição de $\mathrm{C}$ por $\mathrm{T}$ no exon 7 , é capaz de produzir somente $10 \%$ de proteína SMN biologicamente ativa $^{3,4,7}$. Os outros $90 \%$ correspondem a uma proteína instável, sendo rapidamente degradada e portanto, incapaz de manter a função dos neurônios motores ${ }^{2,3}$.

O segmento do genoma onde se localiza o gene da doença, 5q11.2-q13.3, é instável, o que facilita a ocorrência de mutações diferentes no mesmo lócus ${ }^{19,20}$. A AME é, portanto, uma doença em que ambos os sexos são afetados igualmente e que, para aparecer, é necessária a presença do defeito genético em suas duas cópias do gene ${ }^{21}$.

Um ponto importante a ser destacado é o fato de que a quantidade de cópias intactas de SMN-2 é determinante para a severidade da doença $a^{3,5,14}$.

Todo paciente com AME possui pelo menos uma cópia de SMN-2 normal; assim, cerca de $80 \%$ dos pacientes com AME tipo I tem apenas uma ou duas cópias do gene SMN-2, $82 \%$ dos pacientes com AME tipo II tem três cópias do gene SMN-2 e 96\% dos pacientes com AME tipo III tem três ou quatro cópias do gene SMN-2 ${ }^{21}$. Como cada cópia do gene SMN-2 produz cerca de $10 \%$ de proteína SMN biologicamente ativa, quanto mais cópias normais de SMN-2 estiverem presentes, mais proteína estável é produzida, e menor será a gravidade da doença, ${ }^{3,6}$.

O diagnóstico da doença de Werdnig-Hoffmann é feito com base no quadro clínico, nos antecedentes familiares e em exames como: biópsia muscular, achados de estudos neurofisiológicos e eletroneuromiografia ${ }^{22}$. O teste molecular, além de ser necessário para a confirmação da suspeita clínica, é o único que permite o diagnóstico na fase pré-sintomática e a identificação dos portadores ${ }^{14}$. É realizado através da detecção de marcadores na região do cromossomo $5 \mathrm{q}$, permitindo a análise do gene SMN-1. No diagnóstico diferencial, devem ser consideradas todas as causas de hipotonia infantil e síndromes cerebelares, dentre outras menos frequentes ${ }^{15}$.

Em dezembro de 2016, a US Food And Drug Administrantion (FDA), aprovou o primeiro medicamento para uso em pacientes com AME, o Nusinersen $\left(\operatorname{Spinraza}^{\circledR}\right)^{6}$. Até esse momento, o tratamento era focado somente em medidas de suporte, já que não estavam ainda disponíveis alternativas para melhora da força muscular, capazes de alterar o curso natural da doença $\mathrm{a}^{4}$. Tais medidas incluem principalmente o monitoramento cardiorrespiratório e ventilação pulmonar assistida, quando necessário, além da profilaxia de infecções, fisioterapia, dieta hiperproteica e normocalórica, não esquecendo do acompanhamento psicológico, inclusive aos membros da família ${ }^{23}$.

No momento em que surgir a dificuldade intensa na sucção e deglutição, indica-se a alimentação por sonda e posteriormente, a realização de gastrostomia. O prognóstico é reservado e depende da velocidade de evolução da paralisia e do agravamento da função respiratória ${ }^{10}$.

O Nusinersen é utilizado com o objetivo de conter essa progressão da doença, e vem tendo resultados promissores, com melhora da sobrevida dos pacientes, que recebem o medicamento por via intratecal, através de punção lombar. É um oligonucleotídeo antisentido, que se une ao pré-mRNA (pré RNA mensageiro) do gene SMN2, promovendo a inclusão do exon 7 e tornando-o capaz de produzir a forma completa e funcional da proteína $\mathrm{SMN}^{4,5,7}$.

Os pacientes tratados com Nusinersen apresentaram melhora significativa da atividade motora e força muscular, com uma sobrevida livre de ventilação invasiva significativamente maior do que o esperado na evolução natural da doença ${ }^{5,7}$. No estudo multicêntrico fase 2 , iniciado em 2014 e publicado em 2016, crianças présintomáticas, com diagnóstico genético de AME, que iniciaram o tratamento com Nusinersen antes de 6 semanas de vida, não tiveram necessidade de ventilação mecânica e a grande maioria teve desenvolvimento motor normal ${ }^{24}$.

Atualmente, está em curso o estudo multicêntrico fase 3, para avaliação da segurança do medicamento a longo prazo, eficácia e tolerabilidade ${ }^{4}$. No entanto, devido aos bons resultados já obtidos, a Agência de Medicina Européia recomendou a aprovação do Nusinersen para pacientes com AME na União Européia, e sua comercialização foi autorizada em junho de $2017^{3,6}$. No Brasil, a empresa Biogen, responsável pelo medicamento, fez a submissão de registro junto à Anvisa no dia 28 de abril de 2017. O processo recebeu priorização de análise em 4 de maio e após os trâmites necessários, o registro foi concedido em 25 de agosto de 2017. Assim, é esperado que os pacientes brasileiros com AME tenham acesso ao medicamento com mais facilidade ${ }^{25}$.

A doença de Werdnig-Hoffmann é uma condição clínica grave, com expectativa de vida reduzida, em que a maioria dos pacientes atinge, no máximo, cerca de 2 ou 3 anos de idade, caso nenhuma medida terapêutica ou de suporte seja instituída ${ }^{26}$. Nas últimas décadas, devido aos avanços no suporte ventilatório e nutricional, o prognóstico das crianças com AME tem sido alterado, com possibilidade de sobrevivência mesmo com grave comprometimento motor e respiratório?.

Atualmente, podemos afirmar que a sobrevida dos pacientes com AME tipo I é determinada pela escolha do tratamento: pacientes submetidos a traqueostomia com ventilação mecânica têm uma maior sobrevida do que aqueles que utilizam ventilação não invasiva9. Com a aprovação do primeiro medicamento específico para uso na AME e diante de seus primeiros resultados positivos, a história natural da doença poderá ser alterada, porém como os últimos estudos ainda estão em andamento, o impacto do Nusinersen na sobrevida dos pacientes com AME só poderá ser avaliado nos próximos anos $\mathrm{s}^{4,7}$.

Nos pacientes que evoluem para necessidade de ventilação mecânica, a internação em unidade de terapia intensiva (UTI) é necessária até a estabilização clínica compatível com a doença, e sua assistência deverá ser feita por equipe multidisciplinar ${ }^{27}$. 
Após a estabilização clínica, em alguns casos é possível manter a criança em seu próprio domicílio, mesmo em vigência de ventilação mecânica, desde que seja estabelecido um programa de internação domiciliar (home care) adequado para cada caso ${ }^{28}$.

Segundo Soares et al. ${ }^{27}$ a hipotonia e a atrofia muscular são responsáveis pelo comprometimento respiratório e motor e, nesse aspecto, a fisioterapia destacase na equipe multidisciplinar, por atuar no tratamento dos sinais e sintomas dessa síndrome. Além disso, ela também reduz as complicações musculares, apresentando impacto satisfatório no prognóstico dos pacientes, contribuindo significativamente para a superação da expectativa de vida preconizada pela literatura.

Dados preliminares de estudos eletrofisiológicos que incluem a estimativa de unidades motoras em crianças com AME sugerem que a perda de motoneurônios é mais significante no período pós-natal, para a maioria dos pacientes $^{29}$. Nesse sentido, seria necessária a realização de exames para diagnóstico neonatal; ou até mesmo para a detecção pré-natal da AME, a fim de antecipar o acesso a cuidados médicos especiais, e início do tratamento específico ${ }^{5}$.

Wirth et al. ${ }^{30}$ relatam que, em famílias com risco de ter uma criança com AME, poderia ser oferecido um diagnóstico pré-natal, através da análise de amostras da vilosidade coriônica, que é feita entre a $10^{\mathrm{a}}$ e $12^{\mathrm{a}}$ semana de gestação, ou do fluido amniótico, feito entre a $14^{\mathrm{a}}$ e $16^{\mathrm{a}}$ semana.

A importância do diagnóstico precoce, antes do aparecimento dos primeiros sintomas, é reforçada pelos resultados dos estudos clínicos com o Nusinersen, mostrando que o início do tratamento na fase présintomática é capaz de promover um desenvolvimento motor satisfatório ${ }^{5,7,24}$.

Outras opções de tratamento para a AME estão atualmente em estudo, como é o caso da terapia com células-tronco ${ }^{31}$. Nesse campo, ainda existem vários obstáculos a serem superados pelos pesquisadores, a fim de comprovar sua eficácia. Dentre eles, podemos citar: a produção de uma grande quantidade de motoneurônios diferenciados obtidos a partir das mesmas ${ }^{32}$; a permanência de células parcialmente diferenciadas no sistema nervoso após seu implante; as células devem ter a capacidade de estender seus axônios e criar sinapses; e, por último, todas elas devem resultar em uma recuperação funcional significativa ${ }^{33}$.

Outra modalidade promissora de tratamento é a terapia gênica viral, na qual um vírus (AAV9) é utilizado como vetor para reintroduzir uma cópia funcional do gene SMN-1, por via intravenosa, aumentando os níveis da proteína SMN. Atualmente está em curso um estudo fase 1 com esta forma de tratamento em pacientes com AME tipo $I^{3,5}$.

\section{CONSIDERAÇÕES FINAIS}

A atrofia muscular espinhal tipo I constitui a forma mais grave da doença, com o início dos sintomas por volta dos seis meses de idade. O sinal principal é representado por alteração nas fases do desenvolvimento motor da criança, além do grave comprometimento respiratório, que as tornam, na maioria das vezes, dependentes de ventilação mecânica. A evolução natural da doença é o óbito por volta dos três anos de idade, devido à insuficiência respiratória.

É importante ressaltar, ainda, que essa síndrome tem características semelhantes na maioria dos casos e que evolui de forma grave, mas que o tratamento emergencial e intensivo, com equipe multidisciplinar, pode redefinir e prolongar a sobrevida destes pacientes. Em dezembro de 2016, com a aprovação pelo FDA da primeira medicação específica para o tratamento da AME, o Nusinersen $\left(\right.$ Spinraza $\left.^{\circledR}\right)$, as crianças portadoras da doença de WerdnigHoffmann podem ter uma melhora significativa das funções motoras, com grande impacto em sua sobrevida.

Existem casos de crianças que superam a expectativa de vida estipulada para os portadores dessa síndrome, porém, sem a existência de um tratamento capaz de impedir a progressão da fraqueza e da atrofia muscular, essas crianças sobreviviam somente com assistência ventilatória mecânica, necessitando de uma unidade de terapia intensiva (UTI), ou de um programa de home care adequado, mantidas sob contínuo monitoramento cardiorrespiratório.

O tratamento específico, além da melhora na sobrevida que somente poderá ser avaliada após os resultados dos estudos clínicos em curso, tem o potencial de permitir que os pacientes com AME possam manter suas funções respiratórias, sem necessidade de ventilação mecânica. Mesmo com a persistência de algum grau de comprometimento motor, a não utilização de assistência ventilatória, seja ela invasiva ou não, terá um grande impacto na qualidade de vida dos pacientes.

O diagnóstico precoce, que pode ser realizado no período pré-natal ou neonatal, é essencial para um bom resultado do tratamento, já que pode alterar o curso natural da doença caso seja instituído no período pré-sintomático. Para aqueles pacientes que já possuem comprometimento motor importante, o tratamento de suporte e acompanhamento com equipe multidisciplinar, incluindo fisioterapia motora e respiratória, continua sendo de fundamental importância.

Para as crianças em home care, é importante também incentivar sua interação com o ambiente, o que pode ser feito através de um detector de movimentos oculares, aliado a um software para comunicação. Assim, as crianças com AME tipo I são motivadas a aprender e a se comunicar, prevenindo o atraso cognitivo consequente à falta de estímulo, o que também contribui para uma melhor qualidade de vida, tanto para os pacientes quanto para suas famílias ${ }^{1}$. 
AGRADECIMENTOS: Ao Departamento de Medicina da Universidade do Estado de Mato Grosso - UNEMAT - Cáceres/MT, representado pelos docentes Sandra Coenga de Souza, Sidnei Ribeiro de Andrade e Taisir Mahmudo Karim.

Grau de participação dos autores: Lucas Rossato Chrun - Redação, revisão e tradução; Larissa Rossato Chrun Costa - Orientação, tradução e revisão final; Gilson da Silva Miranda - Redação e revisão; Felipe Monteiro Almeida - Redação, revisão e tradução.

\section{REFERÊNCIAS}

1. Polido JG, Barbosa AF, Morimoto $\mathrm{CH}$, Caromano FA, Favero FM, Zanoteli E, Reed UC, Voos MC. Matching pairs difficulty in children with spinal muscular atrophy type I. Neuromusc Disord. 2017;27(5):419-27. doi.org/10.1016/j. nmd.2017.01.017.

2. Robin V, Griffith G, Carter JPL, Leumann CJ, Garcia L, Goyenvalle A. Efficient SMN rescue following subcutaneous tricyclo-DNA antisense oligonucleotide treatment. Am Soc Gene Cell Ther. 2017;7:81-9. doi.org/10.1016/j. omtn.2017.02.009.

3. Bowerman M, Becker CG, Yáñez-Muñoz RJ, Ning K, Wood MJA, Gillingwater TH, Talbot K, The UK SMA Research Consortium. Therapeutic strategies for spinal muscular atrophy: SMN and beyond. Dis Models Mechan. 2017;10:943-54. doi:10.1242/dmm.030148.

4. Maharshi V, Hasan S. Nusinersen: The first option beyond supportive care for spinal muscular atrophy. Clin Drug Invest. 2017;37(9):807-817. doi 10.1007/s40261-0170557-5.

5. Pascual-Pascual SI, García-Romero M. Posibilidades de tratamiento en la atrofia espinal infantil. Rev Neurol. 2017;64(3):19-24. Disponível em: https://www.neurologia. com/articulo/2017173.

6. Wood MJA, Talbot K, Bowerman M. Spinal muscular atrophy: antisense oligonucleotide therapy opens the door to an integrated therapeutic landscape. Hum Mol Genet. 2017;26(R2):R151-R159. doi:10.1093/hmg/ddx215.

7. Talbot K, Tizzano EF. The clinical landscape for SMA in a new therapeutic era gene. Gene Ther. 2017;24(9):529-33. doi:10.1038/gt.2017.52.

8. Teoh HL, Carey K, Sampaio H, Mowat D, Roscioli T, Farrar M. Inherited paediatric motor neuron disorders: beyond spinal muscular atrophy. Neural Plast. 2017;2017:6509493. https://doi.org/10.1155/2017/6509493.

9. Gregoretti C, et al. Survival of patients with spinal muscular atrophy type 1. Pediatrics. 2013;131(5):1509-14. doi:10.1542/peds.2012-2278.

10. Lima MB, Orrico KF, Moraes APF, Negrão CS. Atuação da fisioterapia na doença de Werdnig-Hoffmann: relato de caso. Rev Neurocienc. 2010;18(1):50-4. Disponível em: http:// revistaneurociencias.com.br/edicoes/2010/RN1801/321\%20 relato $\% 20 \mathrm{de} \% 20$ caso.pdf.

11. Marcondes E, Vaz FAC, Ramos JLA, Okay Y. Pediatria básica. 9a ed. São Paulo: Sarvier; 2003.

12. Daniels G, Pettigrew R, Thornhill A, Abbs S, Lashwood A, O'Mahony F, et al. Six unaffected livebirths following preimplantation diagnosis for spinal muscular atrophy. Mol Hum Reproduct. 2001;7 (10):995-1000. doi: http://dx.doi. org/10.1093/molehr/7.10.995.

13. Murrell DV, Lotze TE, Farber HJ, Crawford CA, Wiemann CM. The experience of families with children with spinal muscular atrophy type I across health care systems. J Child Neurol. 2017;32(11):917-923. doi:10.1177/0883073817716853

14. Feng Y, Ge X, Meng L, Scull J, Li J, Tian X, Zhang T, Jin W, Cheng $\mathrm{H}$, Wang $\mathrm{X}$, Tokita $\mathrm{M}$, et al. The next generation of population-based spinal muscular atrophy carrier screening: comprehensive pan-ethnic SMN1 copy-number and sequence variant analysis by massively parallel sequencing. Genet Med. 2017;19(8):936-944. doi:10.1038/gim.2016.215.

15. Collado-Ortiz MA, Shkurovich-Bialik P, González-De Leo S, Arch-Tirado E. Atrofia espinal tipo I (síndrome de Werdnig-Hoffmann). Reporte de un caso. Cir Ciruj. 2007;75:119-122. Disponível em: http://new.medigraphic. com/cgi-bin/resumen.cgi?IDARTICULO=12104.

16. Araújo APQC, Ramos VG, Cabello PH. Dificuldades diagnósticas da atrofia muscular espinhal. Arq Neuropsiquiatr. 2005;63(1):145-9. doi: http://dx.doi.org/10.1590/S0004282X2005000100026.

17. Lefebvre S, Bürglen L, Reboullet S, Clermont O, Burlet P, Viollet L, et al. Identification and characterization of a spinal muscular atrophy-determining gene. Cell. 1995;80(2):15565. doi: http://dx.doi.org/10.1016/0092-8674(95)90460-3.

18. Taylor JE, Thomas NH, Lewis CM, Abbs SJ, Rodrigues NR, Davies KE, et al. Correlation of SMNt and SMNc gene copy number with age of onset and survival in spinal muscular atrophy. Eur J Hum Genet. 1998;6(4):467-74. doi: http:// dx.doi.org/10.1038/sj.ejhg.5200210.

19. Menéndez A, Hernández C, Cepero F. Estudio clínico, genético y molecular en un paciente con atrofia muscular espinal. Rev Cubana Pediatr. 1998;70(1):48-52. Disponível em: http://bvs.sld.cu/revistas/ped/vol70_1_98/ped09198. $\mathrm{htm}$.

20. Russman BS. Spinal muscular atrophy: clinical classifications and disease heterogeneity. J Child Neurol. 2007;22:946-51. doi: http://dx.doi.org/10.1177/0883073807305673.

21. AAME - Amigos da Atrofia Muscular Espinhal. Raskin S. Genética da atrofia muscular espinhal. Disponível em http: www.atrofiaespinhal.org.

22. Prior TW. Spinal muscular atrophy diagnostics. J Child Neurol. 2007;22(8):952-6. doi: http://dx.doi. org/10.1177/0883073807305668.

23. Moreira FA, Moreira LA, Oliveira TO, Aita MA, Moreira MA, Miranda WA. Paciente com síndrome de WerdnigHoffmann. Arq Med. 2004;29(1):61-2. Disponível em: http:// www.portalnepas.org.br/amabc/article/view/322.

24. Finkel RS, Chiriboga CA, Vajsar J, Day JW, Montes J, De Vivo DC, Yamashita M, Rigo F, Hung G, Schneider E, Norris DA, Xia S, Bennett CF, Bishop KM. Treatment of infantileonset spinal muscular atrophy with nusinersen: a phase 2, open-label, dose-escalation study. Lancet. 2016;388:301726. doi: http://dx.doi.org/10.1016/S0140-6736(16)31408-8. 
25. Brasil. Ministério da Saúde. Agência Nacional de Vigilância Sanitária (ANVISA). Registro de medicamentos - Anvisa concede registro ao medicamento Spinraza. Publicado em 25-08-2017. Disponível em: http://portal.anvisa.gov. br/noticias/-/asset publisher/FXrpx9qY7FbU/content/ anvisa-concede-registro-ao-medicamento-spinraza/219201/ pop_up? 101 INSTANCE_FXrpx9qY7FbU viewMode=print $\&$ _101_INSTANCE_FXrpx9qY7FbU_ languageId $=$ pt $B R$

26. Vasconcellos Jorge MGA, Carrapatoso BC, Fernandes ABS. A Fisioterapia na amiotrofia espinhal progressiva tipo I. Rev Neurocienc. 2013;21(3):402-7. doi: http://dx.doi. org/10.4181/RNC.2013.21.731.6p.

27. Soares JA, Silva NLS, Leite SO, Parisi MT. Fisioterapia e qualidade de vida de paciente com amiotrofia espinal progressiva tipo I - Relato de caso. Arq Cienc Saúde. 2006;13(1):44-7. Disponível em: http://repositorio-racs. famerp.br/racs_ol/vol-13-1/ID\%20159.pdf.

28. Vieira LMN, Silva CAN, Oliveira MS, Pimenta LCA. O impacto do cuidado domiciliar na evolução da síndrome de Werdnig-Hoffmann: relato de caso. Rev Med Minas Gerais.

Artigo recebido em: 16.06 .16

Artigo aceito em: 04.10.17
2012;22(4):458-60. Disponível em: http://rmmg.org/artigo/ detalhes/70.

29. Swoboda KJ, Kissel JT, Crawford TO, Bromberg MB, Acsadi G, D'Anjou G, et al. Perspectives on clinical trials in spinal muscular atrophy. J Child Neurol. 2007;22(8):957-66. doi: http://dx.doi.org/10.1177/0883073807305665.

30. Wirth B, Brichta L, Hahnen E. Spinal muscular atrophy: from gene to therapy. Semin Pediatr Neurol. 2006;13:121-31. doi: 10.1016/j.spen.2006.06.008.

31. Lee H, Shamy GA, Elkabetz Y, Schofield CM, Harrsion NL, Panagiotakos G, et al. Directed differentiation and transplantation of human embryonic stem cell-derived motoneurons. Stem Cells. 2007;25(8):1931-9. doi:10.1634/ stemcells.2007-0097.

32. Nayak MS, Kim YS, Goldman M, Keirstead HS, Kerr DA. Cellular therapies in motor neuron diseases. Biochim Biophys Acta. 2006;1762(11-12):1128-38. doi: 10.1016/j. bbadis.2006.06.004.

33. Oskoui M, Kaufmann P. Spinal muscular atrophy. Neurotherapeutics. 2008;5:499-506. doi: http://dx.doi. org/10.1016/j.nurt.2008.08.007. 